\title{
Soft Computing Techniques for Treating Neural Problem: Dementia Used Throughout the World-Areview
}

\author{
Mr.Girish T.M., Dr.Vivek Kumar Sing \\ (Dept. Of Computer Science,Basaveshwar Science College, Bagalkot, Karnataka, India) \\ (Dept. of Computer Science, Banaras Hindu University, Varanasii, U.P., India)
}

\begin{abstract}
Dementia Furthermore it's A large portion regular manifestation, Alzheimer's disease, will be an intricate confusion that afflicts fundamentally the elderly, influencing an evaluated from claiming 63 million toward the quite a while 2030. Concerning illustration there need aid no successful medicinal services medicines to ceasing dementia, along these lines the social mind perspective assumes a paramount part on treating the disease, on superior figure out which intercessions would viable standard What's more dependable Conclusion measures need aid necessary. Writers in this paper aides will indicate that, same time there is no reasonable agreement once An specific measure that exists, same time scientists need aid shutting for looking into a dependable set about measures with assess the Different parts for dementia health: such as cognition, exercises of Everyday living What's more self-destructive considerations and conduct. In this paper writers attempt should furnish a similar Investigation for dementia consideration made in distinctive countries, for a specific concentrate on Alzheimer's illness. The principle reason for existing may be will provide wellbeing policymakers to a finer understanding of the varieties in distinctive methodologies to treating dementia that exist "around every last one of nations to assistance them exceptional define wellbeing approaches.
\end{abstract}

Keywords: Alzheimer's, Dementia, E.E.G. (Electro Encephalogram), M.R.I. (Magnetic Resonance Imaging), Soft Computing

\section{Introduction}

"What's those distinction the middle of dementia Furthermore Alzheimer's?" We listen that constantly here toward those fisher focal point to Alzheimer's examination framework. It's a normal question, What's more doctors could at times help the perplexity. It might be that Doctors like to utilize those statement "dementia" on the term Alzheimer's could heartless All the more overpowering and terrifying. But, those terms Alzheimer's illness Furthermore dementia might intend two extremely distinctive things.

The national foundation for neurological issue Furthermore stroke characterizes dementia as: "Dementia may be aword to an assembly from claiming indications brought about Eventually Tom's perusing issue that influence the mind. It will be not a particular sickness. Kin with dementia might not have the capacity with think great enough to would ordinary activities, for example, getting dressed or consuming. They might lose their capacity to unravel issues or control their feelings. Their personalities might transform. They might turned into foment alternately perceive things that are not there. ". Dementia What's more its mossy cup oak normal manifestation, Alzheimer's disease, is an intricate jumble that afflicts fundamentally the elderly, influencing an assessed 10 million people in distinctive nations. Those intricacy of the sickness makes treating dementia greatly difficult, directing, including a totally assortment of social what's more health awareness intercessions. Typically, these two parts for dementia consideration are analysed independently. This paper adopts a theoretical model that looks at both sorts for intercessions what's more entryway they cooperate along the dementia forethought continuum.

\section{Survey of literature}

John w. LargenLongitudinal transforms On educated support Memory, What's more perceptual capacities done dementia of the Alzheimer's sort [1] (1984) dementia of the Alzheimer's kind (DAT) need been described as an essential degenerative illness of the focal apprehensive framework which is tricky On onset, enduring Furthermore progressive Previously, nature, What's more includes diffuse areas of the cerebrum. Same time those data-base to neuropsychological examples proceeds will create to single-measurement investigations about DAT, there may be a lack from claiming data accessible concerning those changing Also progressive nature of the issue. Those available contemplate might have been initiated will research deliberately the longitudinal examples of intelligence, verbal and nonverbal memory, Also visual-tactile perceptual working on two aggregations for dat patients.

Methuselah, Gerhard Werner [2] (1987) a master framework for finding On geriatric psychiatry this report card will be In view of the thought that criteria to "successful" analysis contrast for separate restorative specialties. Symptomatic judgment clinched alongside exceptionally unstructured assignment domains will be 
best described as clinical issue detailing with even minded suggestions to intercessions. This idea will be illustrated by the execution of a computer-based symptomatic master framework to geriatric psychiatry. Those system starts for directing those patient's meeting Eventually Tom's perusing the clinician. This is refined by frames from claiming diagnoses which are connected with each syndrome. When again, those tolerant information might make consulted on accomplish ideal matches. The demonstrative project of this report card plans toward simulating this transform.

Roy Lachman [3] (1989) master systems: An cognitive science perspective In this paper, methodologies from claiming incorporating sagacity under a workstation system would portrayed alongside An regular structural engineering for master systems, including decisions from claiming representational What's more inferential strategies. Procedure Also ideas are illustrated toward transactions with a shell produced to graduate instruction and a show learning base for those analysis for feeble dementia. Knowledge-based frameworks What's more customary projects need aid compared for admiration to formalisms employed, applications, system characteristics, methods supplied Eventually Tom's perusing those improvement environment, consistency, certainty, flexibility, Also programmer's viewpoint. Those innovation raises essential inquiries for cognitive brain science concerning learning Furthermore dexterity.

Differential determination about dementia: An examination between those master framework show What's more clinicians [4] (1991) the symptomatic execution of the master framework show might have been compared for that of 85 clinicians over diagnosing 10 patients associated with suffice starting with dementia. A multidisciplinary master council gavea standard analysis Likewise reference to correlation. Those comes about demonstrated that those syndrome What's more etiologic diagnoses aggravated by show were over really end concurrence with the individuals of the master council and that those symptomatic execution about show might have been superior to that of the Normal clinician. The introduce discoveries show that master systems, particularly the individuals inside the domain for complex multidimensional restorative problems, Might be a profitable support done restorative practice.

Christian NøhrArtificial brainpower done drug [5] (1994) the assessment about master symptomatic frameworks - how should evaluate results What's more calibreparameters? Improvement of the calibre over health awareness and the appraisal about wellbeing results for restorative innovations have pulled in an expanding consideration in the execution periods. In this paper 10 late assessment investigations are reviewed should examine whatever degree they reflect the structure, process, and result of the theoretical skeleton. It will be discovered that every last one of assessment investigations concentrate on structure measures. Yet In workstation projects on backing restorative choice making would to a chance to be acknowledged in the arranging procedure of the medicinal services system, the assessment investigations must strive to assess methodology Also conclusions measures too. A proposition to a structure to this sort of exploratory Furthermore evaluative examination is delineated.

Shantala $\mathrm{r}$ Basavappa ;Shobini Rao master framework to dementia / melancholy finding [6] (1996) differential determination for dementia What's more dejection obliges finesse. Father will be a computer-aided master system, which utilization those patient's behavioural, cognitive, passionate symptoms; and comes about neuropsychological appraisal to diagnose sorrow alternately dementia. Based to Turbo Prolong, it utilization profundity principal hunt technique with retrograde look technique. DAD's learning base need three information bases. Issue may be spoke to for the issue decrease strategy utilizing four modules. Comes about from those four modules need aid joined together with provide for a last result which will be that point compared for Realities in the learning base. Father diagnoses those the event on the groundwork of this match. Father might have been approved on four patients' records. DAD's finding matched for those treating groups for each body of evidence.

Gary w. Small, subside v. Rabins, Patricia p. Barry, Neil encountered with urban decay because of deindustrialization, engineering concocted, government lodgi. Buckholtz, Steven t. DeKosky, Steven H. Ferris, Sanford i. Finkel,Lisa p. Gwyther, Zaven encountered with urban decay because of deindustrialization, engineering concocted, government lodgi. Khachaturian, barry d. Lebowitz, thomas d. McRae, john c. Morris, Frances Oakley, Lon s. Schneider, joel e. Streim, trey Sunderland, Linda An. Teri, Larry e. Tune, [7] (1997) finding Furthermore medicine for Alzheimer sickness Furthermore related Disorders-A agreement meeting on the analysis and medicine from claiming Alzheimer infection (AD) Furthermore related issue might have been composed Eventually Tom's perusing those American cooperation to Geriatric Psychiatry, the Alzheimer's Association, and the American Geriatrics the public eye once January 4 Furthermore 5, 1997. Those intended interest group might have been elementary consideration physicians, and distinctive inquiries were tended to. Those panellists listened of the experts' presentations, reviewed their foundation papers, et cetera furnished reactions of the inquiries In view of these materials. Alzheimer malady is progressive What's more irreversible, in any case pharmacologic therapies for cognitive hindrance also non pharmacologic What's more pharmacologic medicines for the behavioural issues connected with dementia camwood upgrade personal satisfaction about term. Psychotherapeutic intercession for relatives may be regularly indicated, similarly as almost a large portion of the sum guardians get discouraged. Medicinal services conveyance on these patients will be divided What's more inadequate, Furthermore progressions for ailment management models need aid 
including anxieties of the framework. New methodologies would require to guarantee patients' right to crucial resources, What's more future Look into ought to further bolstering point should move forward symptomatic what's more restorative viability.

TimoErkinjuntti, Trulsøstbye, RunaSteenhuis, c. Psych., Also Vladimir Hachinski, n Engl j [8] (1997) the impact of different symptomatic Criteria on the pervasiveness of Dementia There would a few broadly utilized sets for criteria to those finding from claiming dementia, Anyway little will be known over their level about understanding What's more their impacts on estimates of the pervasiveness for dementia. Creators analysed 1879 men Furthermore ladies 65 A long-time of age or more seasoned who were selected in the Canada wild rye ponder about wellbeing Also maturing Furthermore computed the extent provided for An analysis about dementia as stated by six regularly utilized arrangement systems: those six order frameworks distinguished different aggregations about subjects Concerning illustration Hosting dementia; main 20 subjects were provided for An analysis for dementia as stated by every last bit six frameworks. Those characterizations In view of those Different frameworks differed little as stated by the patients' age, sex, instructive level, or status with admiration to institutionalization. Those regularly utilized criteria to finding camwood contrast Eventually Tom's perusing an element from claiming 10 in the amount of subjects arranged concerning illustration hosting dementia. Such difference need not kidding meanings for exploration What's more treatment, and additionally to those right about large portions more seasoned persons will drive, aggravate An will, Also handle fiscal undertakings.

Elizabeth García-Pérez, ArturoViolante, Francisco Cervantes-PérezExpert frameworks for requisitions [9] (1998) counterfeit consciousness to Mexico neural networks utilizing neural networks for differential determination from claiming Alzheimer sickness and vascular dementia. Differential determination "around distinctive sorts about dementia, basically the middle of Alzheimer (AD) and vascular dementia (VD), offers great challenges because of the covering "around the symptoms, Also indications exhibited Toward patients anguish these illnesses. A differential determination from claiming advertisement also might be acquired with a $100 \%$ of certainty through those examination about mind tissue (i.e.a cerebral biopsy). This gold test includes an obtrusive technique, alsosubsequently it may be infrequently connected. Furthermore these difficulties, to get a proficient differential determination about promotion and VD is essential, a direct result the restorative medicine necessary Eventually Tom's perusing An tolerant contrasts contingent upon those disease he suffers. In this paper, we investigate the utilization about simulated neural networks engineering will manufacture a machine on aid neurologists throughout those differential determination about promotion Furthermore VD. Our outcomes permit us will recommend that, toward utilizing our neural network, neurologists might enhance their effectiveness in getting a right differential determination from claiming notice Also VD and, additionally, that a few tests help minimal of the diagnosis, and that under a few combinations they make it rather additional was troublesome.

Mesulam, m. -Marsel [10] (2000) standards from claiming behavioural Furthermore cognitive neurology, this book gives a clinically propelled However scientifically guided approach of the living establishments of mankind's mental capacity done wellbeing Furthermore malady. It incorporates legitimate scope about every last one of real regions identified with behavioural neurology, neuropsychology, and Furthermore neuropsychiatry. Each chapter, composed towarda world-renowned master in the applicable area, gives an initial foundation and in addition an up and coming survey of the majority late developments. Clinical significance may be accentuated yet may be set in the connection from claiming cognitive neuroscience, fundamental neuroscience, and utilitarian imaging. Significant cognitive domains for example, such that frontal projection function, consideration Also neglect, memory, language, prosody, complex visual processing, Furthermore object ID number need aid reviewed Previously, point of interest. A thorough Section on behaviouralneuro anatomic gives a foundation for brain-behaviour cooperation's in the cerebral cortex, limbic system, basal ganglia, thalamus, and Also cerebellum. Sections for temporo limbic epilepsy, real psychiatric syndromes, Furthermore dementia furnish in-depth analyses for these neurobehavioral substances and their neurobiological coordinates. This book speaks to a priceless asset to behavioural neurologists, neuropsychologists, neuropsychiatrists, and cognitive What's more fundamental neuroscientists. BIGIO, EILEEN H. LIPTON, ANNE m. YEN, SHU-HUI HUTTON, mike 1. BAKER, matt NACHARAJU, PARIMALA WHITE, charles 1. DAVIES, subside LIN, WENLANG DICKSON, DENNIS w. Diary for Neuropathology \& test Neurology: [11] (2001) frontal projection dementia with novel Tauopathy: sporadic numerous framework Tauopathy with Dementia, creators introduce a novel Tauopathy in a tolerant for a 10-yr history for progressive frontal projection dementia and An negative family history. Examination uncovered gentle decay from claiming frontal Furthermore parietal lobes furthermore extreme decay of the fleeting lobes. There were incidental filamentous tau-positive inclusions, Anyway All the more intriguing were various dissimilar globular neuronal Furthermore glial tau-positive inclusions on both gray Also white is concerned of the neocortex. Influenced subcortical districts incorporated substantianigra, globuspallidus, sub thalamic nucleus, What's more cerebellar dentate nucleus, on a circulation comparable with progressive supranuclear paralysis (PSP), be that without critical going with neuronal reduction or gliosis. Predominantly straight 
filaments were distinguished Eventually Tom's perusing electron microscopy (EM), same time other inclusions were comparative will finger impression figures. No turned ribbons were distinguished. Immuno-EM investigations uncovered that best the filamentous inclusions were made from claiming tau. Immunoblotting from claiming sarkosyl-insoluble tau uncovered 2 major groups of 64 Also $68 \mathrm{kDa}$. Blotting dissection after dephosphorylation uncovered predominantly 4-repeat tau. Succession dissection of tau uncovered that there were no transformations in whichever exons 9-13 alternately those contiguous intronic successions. The exceptional cortical tau pathology in this case for sporadic numerous framework tauopathy with dementia includes another pathologic profile of the range of tauopathies.

C's. Encountered with urban decay because of deindustrialization, engineering imagined, government lodgin Herrmanna, t Arnold, An Visbeck, H. -P Hundemer, H. C's HopfComputers for science and drug [12] (2001)Adaptive recurrence decay of E.E.G. for resulting master framework analysis, we display An mixture framework to programmed examination for clinical schedule E.E.G. including a ghastly Investigation Furthermore an master framework. E.E.G.rude information would converted under the time-frequency Webdomain by those purported versatile recurrence decay. Those coming about recurrence parts are changed over under pseudo-linguistic Realities by means of fuzzification. Finally, a master framework applies typical decides figured Eventually Tom's perusing the neurologist with assess the concentrated E.E.G.offers. The framework detects artefacts, portrays alpha musicality toward frequency, amplitude, and Strength Also after antiquity dismissal detects pathologic moderate movement. The greater part comes about would showsimilarly as semantic terms, numerical qualities Furthermore maps for fleeting extent, providing for a review over those clinical schedule E.E.G.

Wimo, Anders; Winblad, Bengt; Aguero-Torres, Hedda; von Strauss, Eva Alzheimer sickness \&cohorted Disorders: [13] (2003) those extent about dementia event in the reality. In this study, those around the world event about dementia to 2000 and throughout those period 1950-2050 might have been evaluated. Those calculations were In light of around the world demographics of the elderly and age-specific predominance what's more occurrence qualities about dementia, assessed starting with An meta-analysis. To an affectability analysis, distinctive pervasiveness sources were utilized. The overall number for persons with dementia done 2000 might have been evaluated during something like 25 million persons. Practically a large portion of the maniacal persons (46\%) existed for Asia, 30\% Previously, Europe, What's more 12\% done NorthAmerica. Fifty-two percent existed to lesquerella produced districts. Something like $6.1 \%$ of the populace 65 along-time of age furthermore more seasoned endured from dementia (about $0.5 \%$ of the overall populace) What's more $59 \%$ were female. The number about new situations from claiming dementia on 2000 might have been evaluated with make 4. 6 million. The conjecture shown a respectable increment in the number for maniacal elderly from 25 million in the quite a while 2000 with 63 million in 2030 (41 million for lesquerella formed regions) Furthermore on 114 million for 2050 (84 million over lesquerella created regions). On conclusion, the dominant part of maniacal elders live in lesquerella created regions, What's more this extent will increment significantly later on.

Encountered with urban decay because of deindustrialization, innovation developed, government lodgin. Iliffe, k. Kharicha, d. Harari, c. Quick andA. E. Stuck [14] (2004) wellbeing hazard examination for more seasoned people as a rule act utilizing a master system: a pilot study, those aversion for handicap previously, after the fact life will be a major test confronting industrialized social orders. Those introduce paper portrays a preliminary examine of the portability for HRA with British settings. Social adjustment and possibility trying of a far reaching wellbeing danger appraisal questionnaire might have been conveyed out in An solitary one assembly act for 12500 patients, Previously, which 58\% of the enrolled number age-old 65 quite some time and In took an interest in the examine. Eight crazy from claiming 10 respondents in the least ages found the questionnaire not difficult alternately exact straightforward What's more complete, despite more than one-third needed or might need loved support. More than half felt that those period of the questionnaire might have been regarding right, What's more you quit offering on that one respondent done 10 hated exactly inquiries. About the individuals who finished the questionnaire What's more gained tailored, composed wellbeing advancement advice, $39 \%$ furnished reaction with respect to this for remarks that might be utilized for expanding those acceptability about custom-made exhortation. These discoveries have educated a wider exploratory consider by and large act.

Encountered with urban decay because of deindustrialization, innovation developed, government lodgin. Iliffe, j. De Lepeleire, H. Van Hout, g. Kenny, a. Lewis, m. Vernooij-Dassen\& those diadem one assembly agincourt\& mental wellbeing [15] (2005) masters from eight European nations (Belgium, France, the Netherlands, Ireland, Italy, Portugal, spain and the united Kingdom) and the controls from claiming clinical psychology, all practice, geriatric medicine, maturity psychiatry, medicinal sociology, nursing Also voluntary figure association met in 2003 will investigate obstacles with distinguishment for Furthermore light of dementia as a rule act inside Europe. An altered centre assembly procedure might have been utilized within this exploratory methodology. Professionals Furthermore individuals for dementia are captivated for overseeing emotional, social What's more physical risks, settling on unequivocal danger administration a possibly vital part

DOI: 10.9790/0661-1804011824 www.iosrjournals.org $21 \mid$ Page


from dementia consideration. Those limit between generalist and pro administrations may be a specific problem, for great possibility to dysfunctionality. Shame and maturing need aid variably disseminated phenomena both inside What's more between nations.

Von Borczyskowski, Daniel; Wilke, Florian; Martin, Brigitte; Brenner, Winfried; Clausen, Malte; Mester, Janos; Buchert, Ralph [16] (2006) assessment of a new master framework to fully robotized identification of the alzheimer's dementia design Previously, FDG PETFluorodeoxyglucose (FDG) positron emanation tomorrow (PET) will be progressively used to help An finding of Alzheimer's sickness. The point of the introduce investigation might have been to assess another master framework (PALZ) for those fully robotized examination about FDG pet pictures to analysis of the illness. The outcomes of the display examine recommended that the PALZ apparatus gives comparative execution for the identification of the commonplace Alzheimer's sickness design for FDG pet pictures Similarly as an encountered spectator underpinned via SPM. Those PALZ device will be completely automated, not difficult should use, and uncaring of the spatial determination of the PET scanner utilized. Therefore, it need those possibility to broad clinical utilization.

D. Fick RN, a. Kolanowski\& j. Waller [17] (2007) helter skelter predominance for focal sensory system medications over community-dwelling more seasoned mature people for dementia In a three-year periodFew late investigations have investigated the pervasiveness and conclusions for focal sensory system (CNS)-active solution utilize for more seasoned persons for dementia (PWD) who live in the group keeping. Thus, the reason for this examine might have been with depict those wellbeing results and examples about utilization of CNS-active pills to PWD living in the group keeping. This examine illustrates the require with further inspect improper CNS-active prescription use for PWD Furthermore on test non-pharmacologic therapies for the clinical issues that start their utilization for PWD.

P. Angelopoulos, H. Agouridaki, H. Vaiopoulos, e. Siskou, k. Doutsou, v. Costa, Furthermore What's more, the lion's share of Corps parts don't stay in their starting work areas once their comm. I. Baloyiannis Cytokines for alzheimer's sickness and vascular dementia [18] (2008) the levels from claiming interleukin $1 \beta$, interleukin 6, What's more interleukin 10 were raised in the serum about patients for dementia. No statistically huge relationship might have been recorded in the interleukin levels around patients for Alzheimer's sickness Furthermore vascular dementia. Also, no noteworthy connection might have been watched in the interleukin levels in the serum and the seriousness of dementia. However, a huge relationship might have been found between IL-6 what's more tumour corruption factor- $\alpha$ (TNF- $\alpha$ ) levels what's more ageists. Those levels of IL$1 \beta$ Furthermore IL-6 were emphatically corresponded for hypertension, Also IL-2 levels were negatively associated. No correspondence might have been found between depressive manifestations Furthermore levels from claiming cytokines in the serum.

Seong-in Kim, donna j. Betts, Heung-Mi Kim, \&Hyung-Seok Kang [19] (2009) Factual models should estimate level for mental jumble dependent upon a workstation rating system: an provision on dementia utilizing organized mandaeanism drawings the mandaeanism need been generally acknowledged Similarly as a powerful symbolization treatment apparatus to focus a client's passionate alternately mental status Furthermore to treat cohorted issue. This model ordered the level for dementia under a standout amongst two categories, extreme or not, Eventually Tom's perusing speaking to it similarly as a pointer subordinate variable. To both models, the paramount free variables were chose toward stepwise regression, and the relative magnitudes for their impacts were compared Eventually Tom's perusing institutionalized relapse. Those framework might have been found will furnish craftsmanship therapists for suitable data on the phases of a patient's propelling dementia. These systems were additionally dictated on a chance to be pertinent with estimating the level and the seriousness for different mental issue.

Jon s. Coulson [20] (2010), Authored SHHHH: a master framework to the management from claiming customers with VOCALLY disruptive practices in DEMENTIAExpert systems, a subset of simulated intelligence, need been utilized to instructive purposes. Master frameworks hold numerous master learning What's more give acceptable choice backing when masters would not accessible. Those master framework depicted in this article might have been produced to train guardians new to the region from claiming dementia forethought in the administration about vocally disruptive practices.

Lindgren H [21] (2011) restrictions in physicians' learning At surveying dementia sicknesses - an assessment consider of a decision-support system There will be a have to gatherings give devices for those restorative proficient In those perspective about consideration in the evaluation of a suspected dementia ailment. Right on time analysis may be imperative so as will gatherings give fitting consideration so that the illness doesn't result in unnecessary suffice to those tolerant and relatives. DMSS (Dementia oversaw economy What's more help System) is a clinical decision-support framework that gives backing in the finding of a dementia disease, which will be being used done regulated clinical assessment settings in four nations. This paper reports those comes about of assessments finished being used situations Previously, these spots Throughout a period from claiming two a considerable length of time. Information on 218 tolerant instances were gathered Toward 21 Doctors Throughout their utilization of the framework to clinical act. Clinched alongside 50 of the cases the utilization of the framework were also watched and the Doctors were interviewed for 88 situations. Those 
gathered information Furthermore inferences produced toward those framework were investigated. On rundown the results, DMSS offered proper help recognizing those tolerant case, accessible data and the user's aptitudes Furthermore information in the area. However, those effects also enlightened those need for developed and customize backing for those lesquerella gifted doctor in the appraisal for fundamental majority of the data over patients.

Thomas Mazzocco, amiodaroneHussain [22] (2012) novel logistic relapse models to help those analysis about dementia Clinicians often experience challenges in the finding for dementia because of the inalienable unpredictability of the methodology and absence of far reaching symptomatic devices. Different models bring been suggested will give acceptable medicinal choice backing in dementia finding. The point for this investigation will be to enhance on the execution of a late provision from claiming Bayesian faith networks utilizing an elective methodology dependent upon logistic relapse. A pool from claiming 14 variables need been assessed onan example for 164 patients associated with dementia. Interestingly, the methodology dependent upon measurable variables Choice outperformed those model which utilized variables chosen Eventually Tom's perusing space masters in the past study. Further community oriented investigations need aid Notwithstanding required on figure out the ideal approach Also on beat existing confinements forced Eventually Tom's perusing the size of the viewed as test.

Jochen René Thyrian, Markus Wübbeler, Wolfgang HoffmannGeriatric mental wellbeing forethought [23] (2013) to Germany, there have been intercessions under those consideration frameworks formed Furthermore executed. This composition depicts An Choice of these. Around An approach level, the WHO stated co-ordination and consideration oversaw economy as an acceptable objective for their dementia arranging model. Those worldwide ideas would comparative yet the heterogeneity about coordinated circuit forethought models makes correlations and interpretation under separate contexts troublesome. There may be a further require should plainly portray means Furthermore objectives from claiming concepts, depict the intends will attain these Furthermore demonstrate their viability what's more effectiveness. For a reconciliation previously, schedule consideration there may be a have should adjust these ideas broadly furthermore actually regionally.

Tilly Eichler, Jochen René Thyrian, danielFredrich, LeonoreKöhler, dianaWucherer, Bernhard Michalowsky, Adina Dreier What's more Wolfgang hoffmann [24] (2014) the profits of actualizing an electronic Intervention-Management-System (IMS) looking into delivering coordinated dementia mind in the grade forethought setting: an electronic Intervention-Management-System (IMS) need been created What's more actualized should encourage dementia forethought oversaw economy. IMS is a rule-based master choice help supportive network that matches unique tolerant qualities on an electronic learning base. A standout amongst the The greater part critical functionalities of IMS is will help the arrangement of the individual mediation want by deliberately distinguishing unmet necessities Also suggesting those comparing particular intercessions for suggestion of the general professional (GP). Those display Investigation pointed with figure out whether the execution of IMS enhances the ID number of unmet needs and the suggestion from claiming sufficient particular intercessions. Our discoveries provide confirmation that IMS enhances those precise ID number about unmet necessities and the ensuing suggestion for intercessions on location these needs. Those clients assessed IMS Similarly as really supportive Also might want to utilize it to their future worth of effort. However, the usability Might be further enhanced.

\section{Conclusion}

Demographic developments help an expanding extent of elderly Also age-associated sicknesses on industrialized nations overall. Dementia may be a standout amongst those the majority common clinched alongside these. Dementia will foundation developing help necessities to those elderly Furthermore consequently gets to be a soundness figure for the mind framework. Particularly industrialized nations similar to north America, the first parts of the EU or Asia have with get ready to this Furthermore create methodologies to their health awareness frameworks. Concerning illustration for every the reports about worldwide load for neurological issue Estimations What's more Projections study there will be enormous deficiency for neurologist done India Also around those planet. Thus writers might want should create an imaginative it built answer for help doctors previously, rustic regions should increase finesse clinched alongside Neuro science What's more treat patients in master neurologist. This paper plans to overview the delicate registering strategies for treating neural patient's issues utilized for those globe for dementia.

\section{References}

[1]. John W. LargenLongitudinal Changes in Intellectual Memory, and Perceptual Functions in Dementia of the Alzheimer's Type 1984, Vol. 24, No. 3-4, Pages 313-314 Texas Research Institute of Mental Sciences, 1300, Moursund, Houston, Texas, 77030

[2]. Methuselah, Gerhard Werner-An expert system for diagnosis in geriatric psychiatry Computers and Biomedical ResearchVolume 20, Issue 5, October 1987, Pages 477-488 DOI:10.1016/0010-4809(87)90035-8

[3]. Roy LachmanExpert systems: A cognitive science perspective Behavior Research Methods, Instruments, \&ComputersMarch 1989, Volume 21, Issue 2, pp 195-204 
Soft Computing Techniques for Treating Neural Problem: Dementia Used Throughout the World ..

[4]. Differential diagnosis of dementia: a comparison between the expert system EVINCE and clinicians http://dx.doi.org/10.1176/jnp.3.4.398 Volume 3 Issue 4, November 1991, pp. 398-404

[5]. Christian NøhrArtificial Intelligence in MedicineVolume 6, Issue 2, April 1994, Pages 123-135The evaluation of expert diagnostic systems - How to assess outcomes and quality parameters?doi:10.1016/0933-3657(94)90041-8

[6]. Shantala R Basavappa ;Shobini L Rao Expert system for dementia / depression diagnosis.NIMHANS Journal. 1996 Apr; 14(2): 99106 Record Identifier: NM000056

[7]. Gary W. Small, Peter V. Rabins, Patricia P. Barry, Neil S. Buckholtz, Steven T. DeKosky, Steven H. Ferris, Sanford I. Finkel, Lisa P. Gwyther, Zaven S. Khachaturian, Barry D. Lebowitz, Thomas D. McRae, John C. Morris, Frances Oakley, OTR; Lon S. Schneider, Joel E. Streim, Trey Sunderland, Linda A. Teri, Larry E. Tune, October 22, 1997 Diagnosis and Treatment of Alzheimer Disease and Related Disorders: Consensus Statement of the American Association for Geriatric Psychiatry, the Alzheimer's Association, and the American Geriatrics SocietyJAMA. 1997;278(16):1363-1371. doi:10.1001/jama.1997.03550160083043

[8]. TimoErkinjuntti, M.D., Ph.D., Trulsøstbye, M.D., M.P.H., RunaSteenhuis, Ph.D., C.Psych., and Vladimir Hachinski, M.D., D.Sc.(Med.) N Engl J Med 1997; The Effect of Different Diagnostic Criteria on the Prevalence of Dementia 337:16671674December 4, 1997DOI: 10.1056/NEJM199712043372306

[9]. Elizabeth García-Pérez,ArturoViolante, Francisco Cervantes-PérezExpert Systems with ApplicationsVolume 14, Issues 1-2, January-February 1998, Pages 219-225Artificial Intelligence in MexicoNeuralnetworksUsing neural networks for differential diagnosis of Alzheimer disease and vascular dementiadoi:10.1016/S0957-4174(97)00076-6

[10]. Mesulam, M.-Marsel (Ed)Database:Principles of behavioral and cognitive neurology (2nd ed.).PsycINFO[ Book; Edited Book ]New York, NY, US: Oxford University Press. (2000). xviii 540 pp.

[11]. BIGIO, EILEEN H. MD; LIPTON, ANNE M. MD, PhD; YEN, SHU-HUI PhD; HUTTON, MIKE L. PhD; BAKER, MATT BSc; NACHARAJU, PARIMALA PhD; WHITE, CHARLES L. III MD; DAVIES, PETER PhD; LIN, WENLANG PhD; DICKSON, DENNIS W. MDJournal of Neuropathology \& Experimental Neurology:April 2001 - Volume 60 - Issue 4 - p 328341 ArticlesFrontal Lobe Dementia With Novel Tauopathy: Sporadic Multiple System Tauopathy With Dementia

[12]. C.S Herrmanna, T Arnold,AVisbeck,H.-P Hundemer,H.CHopfComputers in Biology and MedicineVolume 31, Issue 6, November 2001, Pages 407-427doi:10.1016/S0010-4825(01)00017-8Adaptive frequency decomposition of EEG with subsequent expert system analysis

[13]. Wimo, Anders; Winblad, Bengt; Aguero-Torres, Hedda; von Strauss, EvaAlzheimer Disease \& Associated Disorders:April 2003 Volume 17 - Issue 2 - p 63-67 ArticleThe Magnitude of Dementia Occurrence in the World

[14]. S. Iliffe, K. Kharicha, D. Harari, C. Swift and A. E. Stuck Health risk appraisal for older people in general practice using an expert system: a pilot study Article first published online: 20 DEC 2004 DOI: 10.1111/j.1365-2524.2005.00525.Issue Health\& Social Care in the Community Volume 13, Issue 1, pages 21-29, January 2005

[15]. S. Iliffe, J. De Lepeleire, H. van Hout, G. Kenny, A. Lewis, M. Vernooij-Dassen\& The Diadem Group Aging \& Mental Health Volume 9, Issue 1, 2005 DOI:10.1080/13607860412331323791pages 1-6Publishing models and article dates explainedReceived: 1 Jun 2003Accepted: 2 Feb 2004Published online: 19 Oct 2010

[16]. von Borczyskowski, Daniel; Wilke, Florian; Martin, Brigitte; Brenner, Winfried; Clausen, Malte; Mester, Janos; Buchert, Ralph Evaluation of a new expert system for fully automated detection of the Alzheimer's dementia pattern in FDG PET DOI: 10.1097/01.mnm.0000230078.25609.2b Nuclear Medicine Communications:September 2006 - Volume 27 - Issue 9 - pp 739-743

[17]. D. Fick RN, PhD, A. Kolanowski\& J. WallerVolume 11, Issue 5, 2007High prevalence of central nervous system medications in community-dwelling older adults with dementia over a three-year periodDOI:10.1080/13607860601086629pages 588-595

[18]. P. Angelopoulos, H. Agouridaki, H. Vaiopoulos, E. Siskou, K. Doutsou, V. Costa, and S. I. BaloyiannisCytokines in Alzheimer's Disease and Vascular Dementia2008, Vol. 118, No. 12 , Pages 1659-1672 (doi:10.1080/00207450701392068)

[19]. Seong-in Kim, PhD, Donna J. Betts, PhD, Heung-Mi Kim, MA, Hyung-Seok Kang, BAVolume 36, Issue 4, September 2009, Pages 214-221 Statistical models to estimate level of psychological disorder based on a computer rating system: An application to dementia using structured mandala drawings doi:10.1016/j.aip.2009.03.002

[22]. Jon S. Coulson SHHHH: AN EXPERT SYSTEM FOR THE MANAGEMENT OF CLIENTS WITH VOCALLY DISRUPTIVE BEHAVIORS IN DEMENTIA DOI: 10.1080/036012700407875 pages 401-408 Published online: 11 Nov 2010

[23]. Lindgren H [2011, 169:120-124] Limitations in physicians' knowledge when assessing dementia diseases - an evaluation study of a decision-support system. (PMID:21893726) Type: Journal Article, Multicenter Study

[24]. Thomas Mazzocco, Amir HussainNovel logistic regression models to aid the diagnosis of dementia Expert Systems with Applications Volume 39, Issue 3, 15 February 2012, Pages 3356-3361 doi:10.1016/j.eswa.2011.09.023

[25]. Jochen René Thyrian, Markus Wübbeler, Wolfgang HoffmannGeriatric Mental Health CareVolume 1, Issue 3, September 2013 , Pages 67-71ReviewInterventions into the care system for dementia

[26]. Tilly Eichler, Jochen René Thyrian, Daniel Fredrich, LeonoreKöhler, Diana Wucherer, Bernhard Michalowsky, Adina Dreier and Wolfgang HoffmannThe benefits of implementing a computerized Intervention-Management-System (IMS) on delivering integrated dementia care in the primary care settingInternational Psychogeriatrics / Volume 26 / Issue 08 / August 2014, pp 13771385DOI: http://dx.doi.org/10.1017/S1041610214000830 (About DOI), Published online: 09 May 2014 Copyright $\odot$ International Psychogeriatric Association 2014 